\title{
Contents to Volume 132 (2002)
}

Volume 132 No. 1, May (II) 2002

Alain Kraus: Une question sur les équations $x^{m}-y^{m}=R z^{n}$ (A Question on the Equations $x^{m}-y^{m}=R z^{n}$ )

Phùng Hồ Hải: An Embedding Theorem for Abelian Monoidal Categories

Changyu Xia: Some Applications of Critical Point Theory of Distance Functions on Riemannian Manifolds

François Laubie, Abbas Movahhedi et Alain Salinier: Systèmes dynamiques non archimédiens et corps des normes (Non-Archimedean Dynamic Systems and Fields of Norms)

Alexandra Shlapentokh: Diophantine Undecidability of Function Fields of Characteristic Greater than 2, Finitely Generated over Fields Algebraic over a Finite Field

\section{Volume 132 No. 2, June 2002}

E. Getzler: Euler Characteristics of Local Systems on $\mathcal{M}_{2}$

Yann Bugeaud: Linear Forms in two $m$-adic Logarithms and Applications to Diophantine Problems

Haibao Duan: Self-maps of the Grassmannian of Complex Structures

Alexandru Ioan Badulescu with an appendix by Paul Broussous: Un théorème de finitude ( $A$ Theorem of Finitude)

Atsushi Moriwaki: Nef Divisors in Codimension One on the Moduli Space of Stable Curves

Victor Rotger: On the Group of Automorphisms of Shimura Curves and Applications 
Volume 132 No. 3, July 2002

Eknath Ghate: Adjoint $L$-Values and Primes of Congruence for Hilbert Modular Forms

Viktor Ostrik: Cohomology of Subregular Tilting Modules for Small Quantum Groups

Ngaiming Mok: Characterization of Certain Holomorphic Geodesic Cycles on Quotients of Bounded Symmetric Domains in Terms of Tangent Subspaces

Soo Teck Lee and Hung Yean Loke: Degenerate Principal Series Representations of $\mathrm{U}(p, q)$ and $\operatorname{Spin}_{0}(p, q)$

I. Dolgachev, M. Mendes Lopes and R. Pardini: Rational Surfaces with Many Nodes

$349-363$

Author Index, Volume 132 (2002) 